\title{
Static and dynamic critical properties of the quasi-two-dimensional antiferromagnet $\mathrm{MnPS}_{3}$
}

\author{
A. R. Wildes* \\ Institut Laue-Langevin, 6, rue Jules Horowitz, BP 156, 38042 Grenoble Cedex 9, France \\ H. M. Rønnow and B. Roessli \\ Laboratory for Neutron Scattering, ETH Zürich and Paul-Scherrer Institute, CH-5232 Villigen PSI, Switzerland \\ M. J. Harris \\ ISIS facility, Rutherford Appleton Laboratory, Didcot, Oxon OX11 OQX, United Kingdom \\ K. W. Godfrey \\ Clarendon Laboratory, University of Oxford, Parks Road, Oxford OX1 3PU, United Kingdom \\ (Received 20 March 2006; revised manuscript received 21 June 2006; published 20 September 2006)
}

\begin{abstract}
A comprehensive study of the static and dynamic critical properties of the quasi-two-dimensional antiferromagnet $\mathrm{MnPS}_{3}$ is presented. The relatively large spin $S=5 / 2$ of the Mn ions ensure predominantly classical behavior, and the compound is believed to be a good example of a Heisenberg-type system on a honeycomb lattice. The sample has been measured using a variety of neutron-scattering techniques and instrumentation. The results are compared to the three-dimensional (3D) Heisenberg model, the two-dimensional classical $\mathrm{O}(3)$ rotator model, the two-dimensional $X Y$ model, and the related two-dimensional anisotropic Heisenberg model. The critical properties appear to be best described by the last model almost entirely over the measured temperature range, except just below the Néel temperature where the critical behavior seems to be 3D. There are some discrepancies, particularly in the rescaling of the spin stiffness and the energy widths of the structure factor. A possible explanation for the $X Y$-like behavior is offered, involving the magnetic anisotropy in the system and its influence on the Hamiltonian.
\end{abstract}

DOI: 10.1103/PhysRevB.74.094422

PACS number(s): 75.30.Kz, 75.40.-s, 78.70.Nx

\section{INTRODUCTION}

The physics of critical phase transitions has been driven by theories of magnetism. The magnetic order-disorder phase transitions are often critical in nature and, as the magnetic exchange Hamiltonian is relatively simple, developing a theory to describe a magnetic phase transition is often tractable and sometimes analytically solvable. This is particularly so when the system has less than three dimensions where, when the problem cannot be solved analytically, recent advances in computing power allow ab initio calculations to be performed on large arrays of magnetic moments and the critical dynamics to be analyzed.

To date, the majority of both theoretical and experimental studies on two-dimensional (2D) magnetic systems have been on square lattice compounds. This is understandable, as there are many real compounds that are, to a good approximation, two-dimensional square lattice systems, including highly researched compounds, such as superconducting cuprates and the colossal magnetoresistive manganates. In contrast, less effort has been spent on the study of 2D magnetic systems on a honeycomb lattice. The hypothesis of universality states that the critical behavior is determined by the dimensionality of the system, the symmetry of the order parameters, and the length scale of the forces; thus the conclusions of the extensive studies on square lattice systems should be equally applicable to a honeycomb lattice. It is of interest to measure the critical behavior of honeycomb lattice systems, not least as a check on the hypothesis of universality.

Perhaps one of the reasons why honeycomb lattice systems have not been so heavily investigated is that there ap- pear to be relatively few examples of compounds that might have model behavior. The $\mathrm{Ba}_{2}\left(X \mathrm{O}_{4}\right)_{2} \quad(M=\mathrm{Co}, \mathrm{Ni} ; X$ $=\mathrm{P}$, As) family of compounds is a rare example. ${ }^{1}$ These compounds have attracted interest independently from their honeycomb structure, since they are one of the few known examples of systems that show model $X Y$-like behavior, where the magnetic moments are confined to the plane. Recently, experiments on $\mathrm{BaNi}_{2}\left(\mathrm{VO}_{4}\right)_{2}$ have also been published., ${ }^{2,3}$ The compound has the same crystal structure as $\mathrm{Ba} M_{2}\left(\mathrm{XO}_{4}\right)_{2}$, and it is suggested that this compound is an even better example of an $X Y$ system, with electron-spinresonance experiments, showing evidence of a KosterlitzThouless phase transition. ${ }^{3}$

The $M \mathrm{P} X_{3}(M=\mathrm{Mn}, \mathrm{Fe}, \mathrm{Co}, \mathrm{Ni} ; X=\mathrm{S}, \mathrm{Se})$ family of compounds are also good candidates for $2 \mathrm{D}$ honeycomb magnets. ${ }^{4,5}$ The magnetic transition metal atoms lie in the $a b$ planes, which are held together by weak van der Waals forces. The coupling between the planes is therefore weak both magnetically and atomically. Of this family, $\mathrm{MnPS}_{3}$ would seem to be the simplest to understand. It has a monoclinic structure, space group $C 2 / m$, with lattice parameters $a=6.077 \AA, b=10.524 \AA, c=6.796 \AA$, and $\beta=107.35^{\circ}{ }^{6}$ The manganese atoms have valence $(2+)$, thus having a halffilled $3 d$ shell and spin $S=5 / 2$. Classical theory should therefore be adequate to describe the spin dynamics, and orbital contributions should not exist. The magnetic susceptibility is isotropic at high temperatures, ${ }^{7,8}$ and consequently the magnetic Hamiltonian is believed to be Heisenberg-type. An ideal 2D Heisenberg magnet should not order at a finite temperature; ${ }^{9}$ however, $\mathrm{MnPS}_{3}$ does order antiferromagneti- 


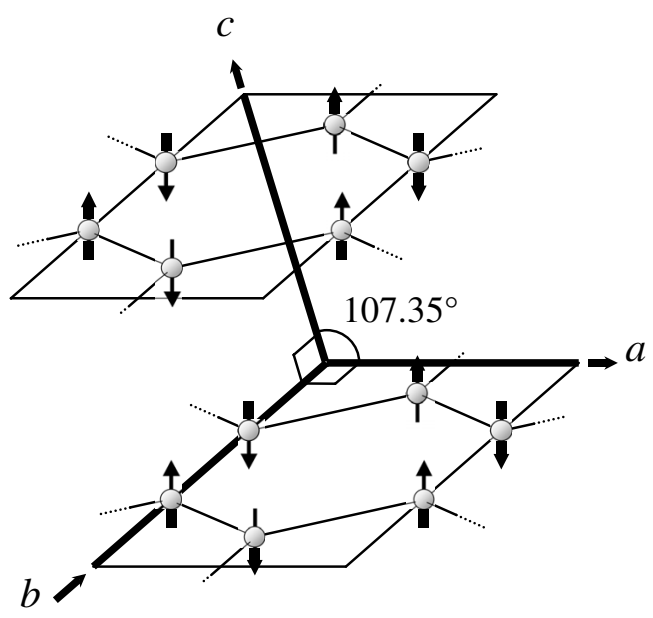

FIG. 1. Schematic showing the magnetic structure of $\mathrm{MnPS}_{3}$. The magnetic moments, given by $S=5 / 2$, point normal to the $a b$ planes.

cally below a Néel temperature of $T_{N}=78 \mathrm{~K}$. The structure of the ordered state was established by neutron diffraction, ${ }^{10}$ with each manganese atom being antiferromagnetically coupled with its nearest neighbors in the plane. The moments point normal to the planes, and there is ferromagnetic coupling between the planes. Figure 1 shows a schematic of the magnetic structure.

Theory shows that a dipole-dipole anisotropy, if strong enough, would be sufficient to induce long-ranged order in a 2D Heisenberg antiferromagnet. ${ }^{11,12}$ Calculations using the theory estimated the dipolar anisotropy in $\mathrm{MnPS}_{3}$ to be strong enough to create a gap in the spin-wave dispersion surface at the Brillouin zone center of $0.7 \mathrm{meV} .{ }^{12}$ A Néel temperature can also be derived using the theory, which for $\mathrm{MnPS}_{3}$ was calculated to be $T_{N}=73 \mathrm{~K}$. The presence of significant dipolar anisotropy would lift the degeneracy of the two spin-wave branches present in the antiferromagnetic phase. The spin-wave dispersion surface has been measured, showing a gap of $0.499 \pm 0.003 \mathrm{meV}$ and, within instrument resolution, degenerate spin-wave branches. ${ }^{13}$ The gap could be modeled by including an easy-axis term in the Hamiltonian, whose size was estimated to be $g \mu_{B} H_{A}$ $=0.0086 \pm 0.0009 \mathrm{meV}$. The dipolar anisotropy was therefore judged to be insufficiently strong to cause long-ranged order.

Even a weak interplanar exchange coupling will lead to a $3 \mathrm{D}$ ordering. As temperature is lowered, the in-plane magnetic correlation length $\xi$ diverges. The area of the correlated regions amplifies the interplanar exchange, $J^{\prime}$. The system will order at the temperature where this energy scale surpasses thermal fluctuations, $T_{N} \sim J^{\prime} \xi^{2}$. A small spin-wave dispersion between the layer planes is present in $\mathrm{MnPS}_{3}$, and the interplanar exchange coupling was calculated to be $J^{\prime}$ $=0.0019 \pm 0.0002 \mathrm{meV}$. This small exchange on its own gives a calculated Néel temperature far smaller than the measured $T_{N}=78 \mathrm{~K}$. Long-ranged ordering is therefore believed to result from a combination of the anisotropy with the weak, interplanar coupling.

The dispersion in the $a b$ plane could be modeled if up to the third nearest neighbors were included. The exchange pa- rameters for the first, second, and third nearest neighbors in the $a b$ plane were $J_{1}=-0.77 \pm 0.09, J_{2}=-0.07 \pm 0.03$, and $J_{3}$ $=-0.18 \pm 0.01 \mathrm{meV}$, respectively. ${ }^{13}$

Initial experiments to measure the critical properties of $\mathrm{MnPS}_{3}$ found some unusual behavior. While the Hamiltonian is believed to be Heisenberg-type, measurements of the magnetic Bragg peak intensity showed that the sublattice magnetization decreased with temperature in a manner that suggested $X Y$-like behavior. ${ }^{13}$ This was further emphasized by the discovery that a Kosterlitz-Thouless model could successfully be applied to the magnetic correlation length above $T_{N}{ }^{14}$ This is particularly interesting, considering the recent observations of $X Y$-like behavior in other 2D honeycomb systems, ${ }^{3}$ potentially hinting at a more general trend.

$\mathrm{MnPS}_{3}$ is therefore quasi-2D, with a weak interplanar exchange and a weak anisotropy that appears to be a combination of both dipolar (out-of-plane) and single-ion (in-plane) contributions. It represents a system that is only slightly removed from the currently derived universality classes and their associated dynamical behavior. It is therefore interesting to compare and see which, if any, of the models currently in the literature correspond. The critical dynamics of $\mathrm{MnPS}_{3}$, therefore, has been comprehensively measured using neutron scattering techniques. Measurements of the temperature behavior of the widths, amplitudes, and renormalized energies of the inelastic scattering at various points in the Brillouin zone above $T_{N}$ are grouped together with measurements of the magnetization, static correlation length, and static amplitude. The data are compared with the theories for a 2D classical $\mathrm{O}(3)$ rotator (CLRM) model, which holds for a Heisenberg-type system, for a two-dimensional $X Y(2 \mathrm{D} X Y)$ model, and the closely related two-dimensional anisotropic Heisenberg $(2 \mathrm{DAH})$ model. Very close to $T_{N}$ the data are compared with the theory of a three-dimensional Heisenberg (3DH) model. An explanation for the dynamical critical behavior of $\mathrm{MnPS}_{3}$ is offered in Sec. V.

\section{MODELS FOR DYNAMICAL SCALING}

The theories for critical phenomena have been summarized in a number of reviews and books (e.g., Refs. 15-17). The theory generally establishes a range of scaling relationships between important physical quantities (e.g., magnetization, correlation length, susceptibility) and the independent variables of temperature and the external magnetic field. The scaling relationships correspond to the nature and dimensionality of the Hamiltonian and can have quite different functional forms.

\section{A. The Heisenberg model in three dimensions (3DH)}

Being quasi-2D, it is expected that a $3 \mathrm{D}$ model will only be applicable to $\mathrm{MnPS}_{3}$ at $T \sim T_{N}$. For $T<T_{N}$, the critical exponent for the sublattice magnetization, $M$, of the $3 \mathrm{DH}$ is, on average, $\beta \sim 0.37^{17}$ and the characteristic frequencies $\gamma_{q}$ at reduced scattering vector $q$ will renormalize according to the relation ${ }^{15}$

$$
\gamma_{q} \propto \xi^{-1 / 2} q
$$

where $\xi$ is the magnetic correlation length. 
Above $T_{N}, \xi$ will decrease with reduced temperature as a power law with exponent $\nu \sim 0.71 .{ }^{17}$ Propagating spin waves cease to exist and are replaced by quasielastic spin fluctuations with a width, at a sufficiently small reduced scattering vector, of $\gamma_{q \sim 0}$. This width will vary with the correlation length according to the relation

$$
\gamma_{q \sim 0} \propto \xi^{-3 / 4} .
$$

\section{B. The two-dimensional classical lattice $\mathrm{O}(3)$ rotator model (CLRM)}

A 2D Heisenberg model can only have long-ranged order at $T=0 \mathrm{~K} ;{ }^{9}$ however, the model allows for a wide temperature range with critical fluctuations at sufficiently low temperatures. Scaling relationships for these fluctuations have been derived by mapping them onto the classical rotator $\mathrm{O}$ (3) model or classical lattice rotator model (CLRM). ${ }^{18-20}$ The relations have been applied with some success to measurements on 2D antiferromagnets, ${ }^{21,22}$ all of which were square lattice systems. As a potential example of a quasi-2D Heisenberg antiferromagnet, the model should also apply to $\mathrm{MnPS}_{3}$ at temperatures above $T_{N}$ where $3 \mathrm{D}$ correlations become negligible.

Two parameters are necessary for the theory to predict both the static and the dynamic critical behavior of the CLRM: the spin stiffness, $\rho_{S}\left(=J S^{2}\right.$ in two dimensions); and the zero-temperature spin-wave velocity, $c(=2 \sqrt{2} J S a / \hbar$ in $2 \mathrm{D}$, where $a$ is the lattice parameter). These quantities can be calculated for $\mathrm{MnPS}_{3}$ from the measured spin wave dispersion curve. ${ }^{13}$ Using the nearest-neighbor interaction $\left(\left|J_{1}\right| / k_{\mathrm{B}}=8.9 \mathrm{~K}\right)$ gives a spin stiffness of $\rho_{S} / k_{\mathrm{B}} \equiv 56 \mathrm{~K}$, and the gradient of the spin-wave dispersion along the $[0, k, 0]$ direction gives a spin-wave velocity of $c / k_{\mathrm{B}}=346 \mathrm{~K} \AA$. Quantum corrections are often applied to $\rho_{S}$; however, they are negligible for $S=5 / 2$ systems and will be ignored.

Spin waves can exist in the CLRM at low temperatures; however, they will be heavily damped. ${ }^{19}$ The dynamic orderparameter correlation function is then written ${ }^{18}$

$$
S(q, \omega)=\gamma_{q \sim 0}^{-1} S(q) \Phi\left(q \xi, \omega \gamma_{q \sim 0}^{-1}\right) .
$$

The equal-time correlation function, $S(q)$, has a Lorentzian form $^{18}$ with the width being inversely proportional to the correlation length $\xi$. The correlation length is expected to scale with temperature as ${ }^{20}$

$$
\xi=\frac{e}{8} \frac{\hbar c}{2 \pi \rho_{S}} \exp \left(\frac{2 \pi \rho_{S}}{k_{B} T}\right)\left(1-\frac{k_{B} T}{4 \pi \rho_{S}}+O(T)^{2}\right),
$$

and the amplitude of the equal-time correlation function, $S(q=0)=S_{0}$, is expected to scale with temperature and $\xi$ and $T$ as

$$
S_{0} \propto \xi^{2} T^{2} .
$$

The scaling relation for the quasielastic width is given by ${ }^{18}$

$$
\gamma_{q \sim 0}=c \xi^{-1}\left(k_{B} T / 2 \pi \rho_{S}\right)^{1 / 2}
$$

Renormalization of the spin-wave energies is only important very close to the Brillouin zone center, where it scales with $T$ and $\xi^{18,19}$ The spin-wave damping $\gamma_{q}$ renormalizes with temperature. The scaling relations differ as a function of the wavelengths of the spin waves, with the damping of the long wavelength fluctuations increasing fastest with temperature. ${ }^{19}$

\section{The two-dimensional $X Y$ model (2D $X Y)$}

The 2DXY model constrains the magnetic moments to lie in the plane, and has been applied in the analysis of a number of 2D antiferromagnets, including some with honeycomb lattices. ${ }^{1,3}$ The model predicts a phase transition temperature, $T_{K T}$, associated with the unbinding of spin vortices. All the real systems discovered so far have a $3 \mathrm{D}$ ordering at a temperature just above $T_{K T}$. Over length scales smaller than $L$ $=\sqrt{J / J^{\prime}}$, however, the model predicts that a system will behave in a true $2 \mathrm{D}$ fashion. ${ }^{23}$

The $2 \mathrm{D} X Y$ model has a sublattice magnetization, $M$, with a critical exponent $\beta=0.231 .^{23}$ A previous measurement of the temperature dependence of the magnetization in $\mathrm{MnPS}_{3}$ found a critical exponent $\beta=0.25 \pm 0.01$, very close to that expected for a $2 \mathrm{D} X Y$ model. ${ }^{13}$

The correlation length $\xi$ will scale with temperature according to the equation ${ }^{24}$

$$
\xi=A \exp \left(b_{K T} \sqrt{T_{K T} /\left(T-T_{K T}\right)}\right),
$$

where $A$ and $b_{K T}$ are nonuniversal constants. The length $L$ is calculated from the exchange parameters, which are known for $\mathrm{MnPS}_{3}$, but must also be consistent with the equation ${ }^{23}$

$$
\frac{T_{N}-T_{K T}}{T_{N}}=\frac{b_{K T}^{2}}{\left(\log _{e} L\right)^{2}} .
$$

Propagating spin waves can exist below $T_{K T}$, and the characteristic frequency will scale with temperature and correlation length in a similar manner as Eq. (1), with a slightly different exponent on $\xi .{ }^{15}$ Unlike the CLRM, no propagating spin-wave modes exist above the transition temperature, $T_{K T}$.

\section{The anisotropic two-dimensional Heisenberg model (2DAH)}

The Hamiltonian for the spin dynamics is often written to be anisotropic

$$
H=-J \sum_{\langle i, j\rangle}\left(S_{i}^{x} S_{j}^{x}+S_{i}^{y} S_{j}^{y}+\lambda S_{i}^{z} S_{j}^{z}\right)
$$

where $0 \leq \lambda \leq 1$. In the limit $\lambda=1$, the Hamiltonian becomes isotropic and the dynamics will map onto a Heisenberg model. In the limit $\lambda=0$, there is exchange coupling only between $x$ and $y$ components of the moments, as for the $2 \mathrm{D} X Y$ model. Unlike the 2DXY model, however, the moments are allowed to fluctuate in the $z$ direction; thus the model is more representative of real systems. The transition from CLRM to $2 \mathrm{DXY}$ behavior as a function of $\lambda$ has been expressed analytically. ${ }^{25}$

There have been a large number of theory studies of the 2DAH model. ${ }^{17}$ The critical behavior for $\lambda<1$ falls into the same universality class as the $2 \mathrm{D} X Y$ model; thus the temperature behavior of $M$ and $\xi$ is the same as discussed in Sec. 
II C. The 2DAH generally deals with the thermodynamics of vortices.

The dynamic structure factor, $S(q, \omega)$, can be expressed in the same manner as Eq. (3). Two structure factors are relevant to the 2DAH model. The first, $S^{x x}(q, \omega)$, is derived from the in-plane correlation function, while the second $S^{z z}(q, \omega)$ is derived from the out-of-plane correlations. Above $T_{K T}$, the spin fluctuations become quasielastic. The functional form for $S^{x x}(q, \omega)$ has been calculated to be a squared Lorentzian form, while $S^{z z}(q, \omega)$ takes the form of a Gaussian. ${ }^{26}$

For $T>T_{K T}$, the function $S^{x x}(q)$ is a Lorentzian to the power $3 / 2$ whose amplitude, $S^{x x}(q=0)=S_{0}^{x x}$, will scale as $^{26}$

$$
S_{0}^{x x} \propto \xi^{2} .
$$

The width scales according to the relation ${ }^{26,27}$

$$
\gamma_{q} \propto \xi^{-2}\left(1-\frac{T}{T_{K T}}\right)^{-1 / 4}\left[1+(q \xi)^{2}\right]^{1 / 2} .
$$

The critical dynamics of the 2DAH model has been investigated numerically. ${ }^{28-31}$ Just above $T_{K T}$, the calculations show that propagating spin waves can still exist. The inplane structure factor quickly becomes quasielastic with increasing temperature, but the out-of-plane structure factor will show spin waves to higher temperatures. The importance of the out-of-plane component depends on the value of $\lambda$ in Eq. (9), as this anisotropy determines the orientation of the vortices. It has been calculated that, for a honeycomb lattice, the vortices will develop an out-of-plane component when $\lambda>0.86 .^{30}$

\section{EXPERIMENTS}

The sample used in the experiment was a single crystal made by techniques explained previously. ${ }^{13}$ The sample was aligned such that the scattering plane was spanned by the $[0,0,1]$ and $[0,1,0]$ vectors. $\mathrm{MnPS}_{3}$, with two magnetic atoms per unit cell, has a magnetic propagation vector of $(0,0,0)$ and therefore all magnetic Bragg peaks are also nuclear Bragg peaks. The scattering plane therefore contained the $(0,2,0)$ Bragg peak, which is also one of the strongest magnetic Bragg peaks. The critical scattering rods were expected to be parallel to $[0,0,1]$, thus in the plane of scattering. The sample orientation was therefore suitable for all the critical scattering experiments.

A qualitative examination of the critical scattering was carried out on the PRISMA spectrometer at the ISIS neutron spallation facility, Rutherford Appleton Laboratories, UK. ${ }^{32}$ The time-of-flight instrument was used in diffraction mode with no energy analysis. When used in this configuration, PRISMA is able to quickly detect any diffuse scattering, including rodlike structures. Temperature control was achieved with a helium cryorefrigerator. Measurements were made at three temperatures: $300 \mathrm{~K}$, where $\mathrm{MnPS}_{3}$ is expected to be paramagnetic; $8 \mathrm{~K}$, where the system is antiferromagnetic; and $100 \mathrm{~K}$, approximately equal to the maximum in the magnetic susceptibility.

The critical behavior of the sublattice magnetization and the magnetic correlation length $\xi$ were measured using the
RITA neutron three-axis spectrometer at Ris $\varnothing$ National Laboratories, Denmark. The sample was mounted in a helium cryostat, capable of controlling the temperature of the sample to $< \pm 0.1 \mathrm{~K}$. Higher-order wavelength contamination was suppressed with a velocity selector. The instrument was initially configured with a graphite monochromator and analyzer. The Néel temperature $T_{N}$ was carefully determined by measuring the temperature dependence of the $(0,2,0)$ Bragg peak and was in close agreement with previously published values. Measurements well above $T_{N}$ were used to estimate the nuclear intensity, which was subtracted from the data below $T_{N}$ to determine the magnetic contribution. These measurements permitted an accurate determination of the critical exponent for the magnetization, $\beta$. The analyzer was then removed and the instrument subsequently used in a twoaxis mode with the incident energy fixed at $E_{i}=4.84 \mathrm{meV}$. Collimators of $30^{\prime}$ were put before and after the sample to limit divergence, and the resolution of the instrument was measured by making a reciprocal space map around the $(0,2,0)$ Bragg peak. To measure the temperature dependence of $\xi$, scans were carried out along $(0,2 \pm k, 0.6)$ as a function of temperature above $T_{N}$. These scans were chosen for three reasons. The scan trajectory is perpendicular to the critical scattering rods; the $(0,2,0.6)$ position is relatively free of contamination due to Bragg peaks and their mosaic tails; and at $(0,2,0.6)$ the final wave vector $\mathbf{k}_{F}$ is aligned parallel to the critical scattering rods. The measurements integrate all the scattering along $\hat{\mathbf{k}}_{F}$, limited by the kinematic constraints of the incident neutron energy, and therefore, the scans represent a measurement of the equal-time structure factor, $\int_{-\infty}^{E_{i}} S(q, \omega) d \omega \approx S(q)$. The RITA spectrometer is particularly suitable for this type of measurement as the velocity selector allows flexibility in choosing an appropriate incident energy free of higher order contamination, and the 2D positionsensitive detector allows for a precise estimation of background.

The dynamic critical scattering was measured using the IN12 and IN14 cold neutron three-axis spectrometers at the Institut Laue-Langevin, France. Both instruments were configured with graphite monochromators and analyzers. The sample was mounted in a helium cryostat for temperature control. Higher order contamination was filtered from the scattered beam, using a cooled beryllium filter.

The IN14 spectrometer was used to follow the critical behavior of the dynamics. Two resolution configurations were used. In both configurations the monochromator was curved to focus the beam in the vertical direction and the final wave number $k_{F}$ was $1.55 \AA^{-1}$. When measuring close to $T_{N}$, the instrument was configured with a flat analyzer and $40^{\prime}$ analyzers were placed before and after the sample. This configuration was also used to establish $T_{N}$ from the magnetic intensity of the (020) Bragg peak. Further from the Néel temperature, where the measured intensity became weak, the collimators were removed and the analyzer was curved for horizontal focusing.

The IN12 spectrometer was used to measure the inelastic scattering very close to $T_{N}$. The instrument was used with a vertically curved monochromator and a flat analyzer. Collimators of between $30^{\prime}$ and $60^{\prime}$ were between monochro- 
mator and sample, sample and analyzer, and analyzer and detector. A number of combinations of collimator and choices of $k_{F}$ were chosen as better resolution or intensity was required.

The resolutions of all configurations were measured by scanning around the $(0,2,0)$ Bragg peak and by measuring the energy width of the incoherent scattering. Resolution calculations showed an adequate comparison with the measurements, being within $\sim 10 \%$ of the measured dimensions of the resolution ellipsoids.

\section{RESULTS}

\section{A. Qualitative measurement of the two-dimensional critical scattering}

The neutron diffraction measurements from the PRISMA spectrometer are shown for the three measured temperatures in Fig. 2. The Bragg peaks are clearly seen at positions that can be indexed to give lattice parameters $b=10.52 \AA$ and $c$ $=6.080 \AA$, consistent with the expected values ${ }^{6}$ Also visible are two aluminum powder rings from the sample holder. A number of very small peaks at positions other than those allowed for Bragg peaks show that the crystal is not perfect, but has very small crystallites attached to the large single crystal. Care was taken in all subsequent experiments to choose scans in reciprocal space that are free of these extra peaks.

As expected from the magnetic structure factor, rods are clearly visible along $(0,2, l)$ and $(0,4, l)$, but not along $(0,6, l)$. Traces of the rods are visible at all temperatures; however, they are strongest at $100 \mathrm{~K}$. This is clearly demonstrated in Fig. 3, which shows the scattering along $(0,2, l)$ as a function of temperature. Also shown in Fig. 3 is the scattering along $(0,6, l)$ below and above $T_{N}$. Consistent with the magnetic structure factor, the magnitudes of the $(0,6,0)$ and $(0,6,2)$ Bragg peaks remain unchanged with temperature.

The data in Fig. 3 also appear to show satellites at $(0,2,1 / 3)$ and $(0,2, \overline{1} / 3)$, which are present because the monoclinic structure of $\mathrm{MnPS}_{3}$ is only slightly distorted from being hexagonal with a periodicity of three unit cells along $[0,0,1]$. Similar peaks have been observed and explained in x-ray powder diffraction from $\mathrm{MnPS}_{3} \cdot{ }^{33}$ The peaks are of similar width to the $(0,2,0)$ Bragg peak, and scale with temperature in a similar manner. They are therefore useful as they show the temperature behavior of the $(0,2,0)$ Bragg peak, which is off-scale in Fig. 3; the peaks at 100 and $300 \mathrm{~K}$ are similar in intensity, as the sample is above the Néel temperature and consequently, only nuclear scattering contributes to the Bragg peaks, whereas strong magnetic scattering at $8 \mathrm{~K}$ results in a doubling of the Bragg peak intensities.

\section{B. The critical behavior of the magnetization below $T_{N}$}

Figure 4 shows the magnetic Bragg peak intensity from the $(0,2,0)$ Bragg peak, measured on the RITA spectrometer, as a function of the reduced temperature $\left(1-T / T_{N}\right)$. The data follow a power-law relationship over the entire temperature range, which is consistent with critical scaling. The critical

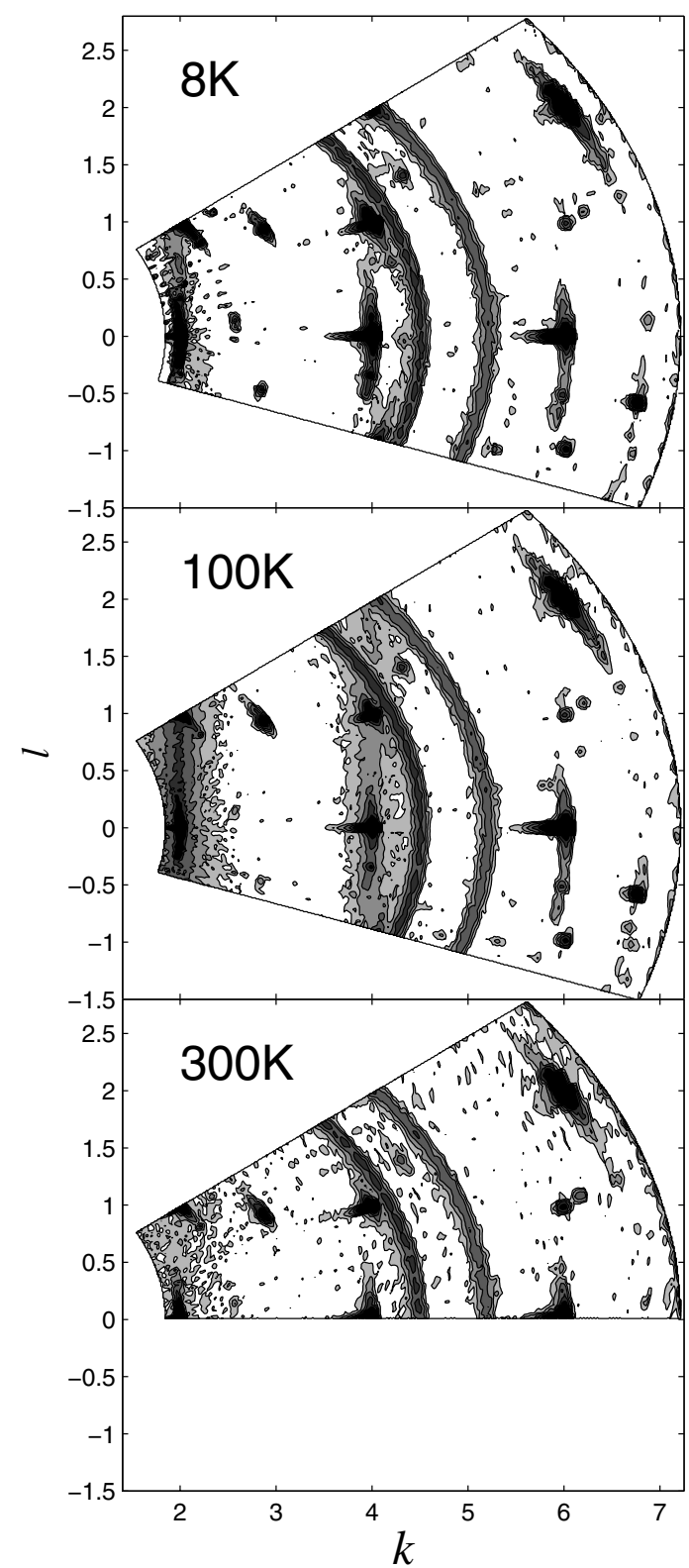

FIG. 2. Neutron scattering intensity in the $b^{*} c^{*}$ plane of $\mathrm{MnPS}_{3}$ below $T_{N}(8 \mathrm{~K})$, at the maximum in the magnetic susceptibility $(100 \mathrm{~K})$ and at room temperature. The data were measured using the PRISMA spectrometer in diffraction mode.

exponent, $\beta$, is shown to be $\beta=0.25 \pm 0.01$ for $\left(1-T / T_{N}\right)$ $>0.03$. This is identical to the value for $\beta$ determined in a previous experiment, ${ }^{13}$ and is close to the signature value for the 2DAH model of $\beta=0.231$. $^{23}$ The previously published data hinted at a change in $\beta$ close to $T_{N}$, although the quality of the data was not sufficient to be certain. The data presented in Fig. 4 are the result of a more careful experiment, and the figure indeed shows that there is a crossover in the critical behavior to $\beta=0.32 \pm 0.01$ for $\left(1-T / T_{N}\right)<0.03$. This change in $\beta$ is consistent with a crossover from two- to threedimensional behavior. ${ }^{16,17}$ 


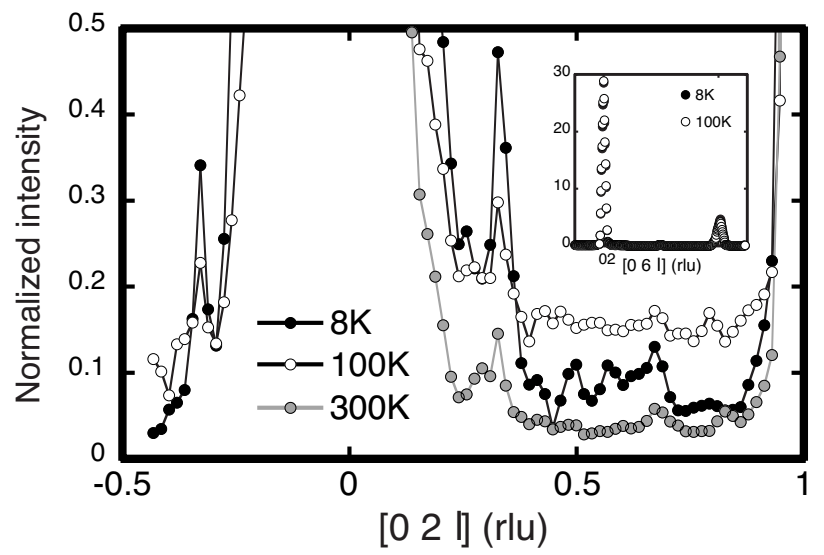

FIG. 3. Extracted intensities along $(0,2, l)$ from Fig. 2 at the three temperatures. The insert shows the extracted intensities along $(0,6, l)$, where the magnetic scattering is zero due to the magnetic structure factor.

The crossover temperature coincides with a change in the rate of decrease of the gap in the spin-wave energies at the Brillouin zone center. ${ }^{13}$ The gap was also observed to decrease as a power law over the full range of temperature. At low temperatures the exponent equaled $0.26 \pm 0.02$, comparable to the value of $\beta$. Spin-wave gaps with the same temperature dependence as the magnetization have been seen before in a variety of $2 \mathrm{D}$ materials. ${ }^{34,35}$ For $T_{N}>T$ $>0.96 T_{N}$, the exponent changed to equal $0.51 \pm 0.02$, which could be understood as the gap varying with the magnetization to the power $\sim 3 / 2$. Similar changes have been discussed in the context of magnetic anisotropy, and related to the Legendre polynomials that dominate in the expansion of the anisotropic energy. ${ }^{36}$ It is plausible that, with the crossover to 3D behavior, different order Legendre polynomials will become dominant, resulting in the observed change in the exponent.

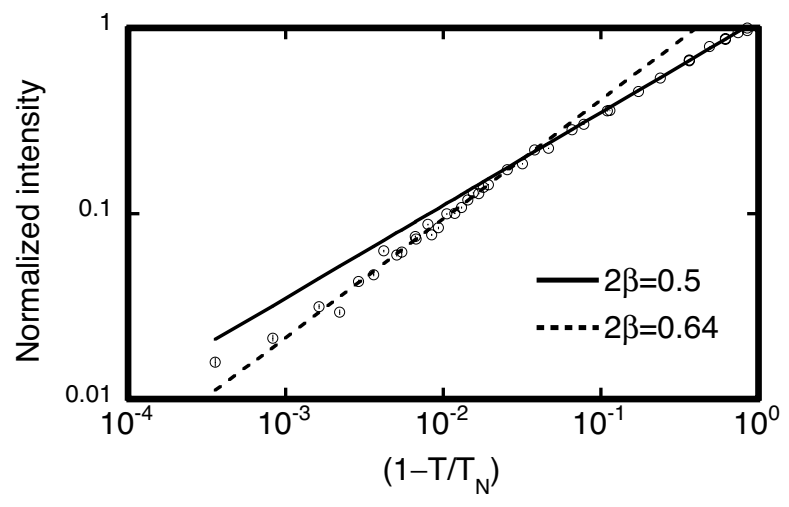

FIG. 4. The temperature dependence of the staggered magnetization, as estimated from the measured intensities of the $(0,2,0)$ Bragg peak. The Néel temperature was found to be $78.6 \mathrm{~K}$. The data have had the nuclear intensity subtracted and are normalized by being divided by the intensity at the lowest temperature. Also shown are fits of power laws to the magnetization, showing a crossover from $\beta=0.25$ to $\beta=0.32$ at $T \sim 0.97 T_{N}$.

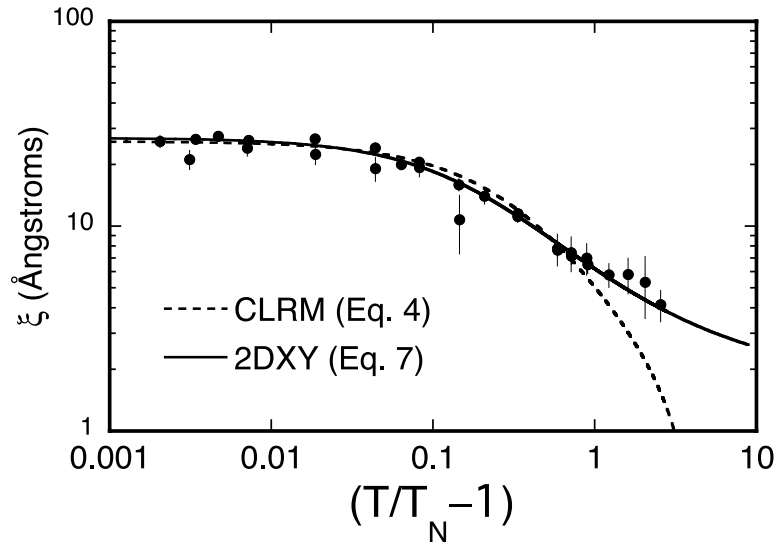

FIG. 5. The temperature dependence of the correlation length above $T_{N}$, as estimated from the fitted widths of the energy integrated critical scattering rods. Also shown are the fits of Eqs. (4) and (7) to the data.

\section{The magnetic correlation length above $T_{N}$}

The temperature-dependent scans through the critical scattering rods, as measured using the RITA spectrometer in two-axis mode, were fitted with Lorentzian functions, convoluted with the full experimental resolution. ${ }^{14}$ The magnetic correlation lengths are shown in Fig. 5. The correlation length $\xi$ falls from $\sim 27.5 \AA$ at $T_{N}$ to $\sim 4.1 \AA$ at $280 \mathrm{~K}$. The data could then be compared to the models in Sec. II. Fits of Eqs. (4) and (7) are also shown in Fig. 5.

Equation (4) fits well to the data close to $T_{N}$; however, at larger temperatures it falls much faster than the data. This is not surprising as the function is expected to be valid only for large correlation lengths. ${ }^{20}$ The fitted parameters gave an amplitude of $e \hbar c /\left(16 \pi \rho_{s}\right)=2.0 \pm 0.3 \AA$ and $\rho_{s} / k_{\mathrm{B}}=35 \pm 2 \mathrm{~K}$. The latter value is considerably smaller than the value expected, $\rho_{S} / k_{\mathrm{B}} \equiv 56 \mathrm{~K}$; thus, the parameters of the CLRM fail to match the physical properties that they represent.

Equation (7) fits well to the experimental data over the entire temperature range with the fitted parameters being $A$ $=1.6 \pm 0.3 \AA, b_{K T}=1.98 \pm 0.01$, and $T_{K T}=55 \pm 4 \mathrm{~K}$. The parameter $b_{K T}$ is a nonuniversal constant, but was initially estimated to be $b_{K T} \sim 1.5,{ }^{24}$ which is not far from the fitted value. The parameters are also consistent with Eq. (8). Inserting values for the measured exchange integrals and the measured $\xi\left(T_{N}\right)=27.5 \AA$ gives $A=1.37 \AA$, close to the fitted value.

The fitted amplitudes, corresponding to $S_{0}$, have been divided by $\xi^{2}$ and are shown in Fig. 6. In the CLRM, Eq. (5) shows that the ratio should scale with $T^{2}$. On the other hand, the ratio is constant with temperature in the 2DAH models, as is shown in Eq. (10). The measured ratio is approximately constant with temperature, further confirming that the 2DAH models better represent the static critical behavior of $\mathrm{MnPS}_{3}$.

\section{The dynamic critical behavior at $q \sim 0$}

Above $T_{N}$, a magnetic Bragg peak becomes a quasielastic rod, as seen in Fig. 2. The quasielastic energy width of the scattering $\gamma_{q \sim 0}$ was measured as a function of temperature 


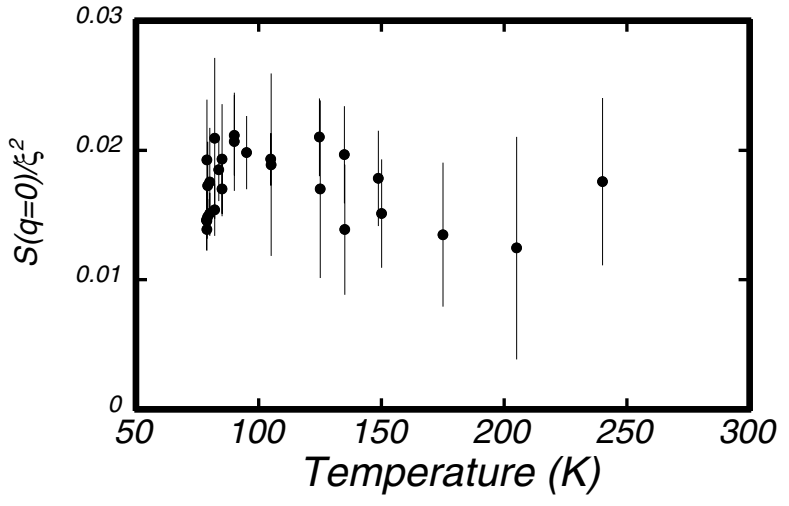

FIG. 6. The amplitude of the critical scattering rods divided by the correlation length squared as a function of temperature, as determined from fitting Lorentzian functions to the data.

by measuring the inelastic scattering at constant $Q=(0,2,1$ $-\varepsilon)$, where $\varepsilon$ is small. Examples of the scattering at three temperatures, measured on IN14, are given in Fig. 7. All the data clearly showed a Gaussian-type peak at zero energy transfer, corresponding to the incoherent scattering, with a broad, quasielastic signal underneath.

The $S(q \sim 0, \omega)$ data were fitted with functions to determine the width, $\gamma_{q}$. Two forms of function were used-one with a Lorentzian form, as suggested by the CLRM, and one with a squared Lorentzian form, as suggested by the $2 \mathrm{DAH}$. At higher temperatures the functions gave fits of equal quality; however, close to $T_{N}$ the Lorentzian function better represented the data. The parameters from both functions varied identically with temperature; thus, only the data from fitting the Lorentzian function will be presented.

The Lorentzian followed from the spectral weight function for critical scattering in the hydrodynamic limit ${ }^{37}$

$$
\mathrm{S}(\boldsymbol{q} \sim 0, \omega)=[\mathrm{n}(\omega)+1] \frac{\omega A_{q \sim 0} \gamma_{q \sim 0}}{\gamma_{q \sim 0}^{2}+\omega^{2}},
$$

where $A_{q \sim 0}$ is the amplitude and $n(\omega)$ is the Bose function, $n(\omega)=1 /\left(1-\exp \left(-\hbar \omega / k_{B} T\right)\right)$. Equation (12) has the correct form for a dynamic structure factor, with finite moments and proper weighting for mode population, and is similar in form to equations used in the analysis of other 2D classical antiferromagnets (e.g., Ref. 21). In fitting the data, the function was convoluted with the resolution of the instrument using a Monte Carlo algorithm, and a Gaussian peak was added to model the incoherent scattering. Examples of the fits are shown in Fig. 7. The widths of the fits $\gamma_{q \sim 0}$ are plotted as a function of temperature in Fig. 8.

As expected, $\gamma_{q \sim 0}$ increases with increasing temperature; however, the nature of the increase appears to be at odds with scaling theory. Scaling theory predicts that $\gamma_{q \sim 0}$ should vary with the correlation length $\xi$, as shown in Eqs. (2), (6), and (11). Figure 8 shows that the widths appear to increase linearly with temperature, rather than varying with $\xi$. Thus the data have been fitted with a straight line, also shown in Fig. 8, with gradient $0.050 \pm 0.001 \mathrm{meV} / \mathrm{K}$ and intercept $-3.8 \pm 0.1 \mathrm{meV}$. That the data do not match the scaling relations predicted by the models is further illustrated in the inset

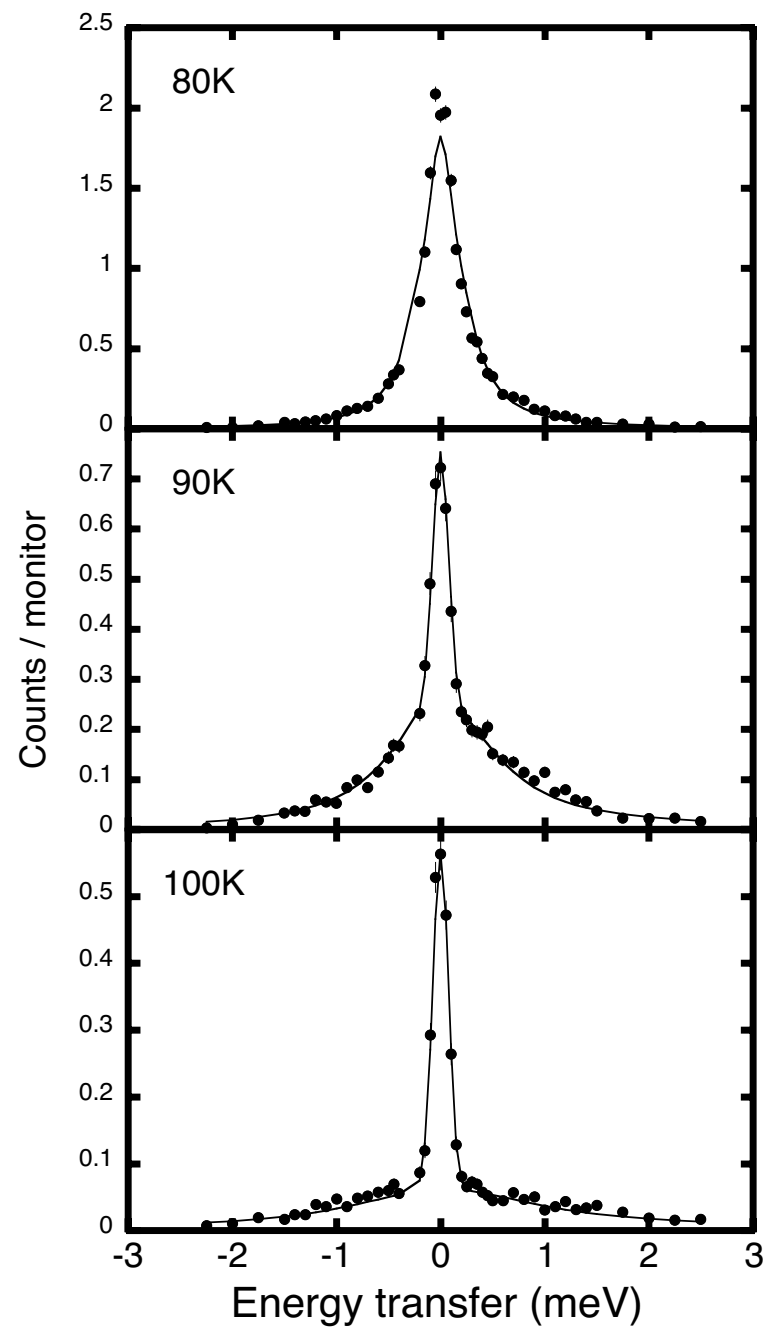

FIG. 7. Examples of inelastic measurements close to the $(0,2,1)$ Bragg peak above $T_{N}$. All the data show a resolutionlimited incoherent peak with quasi-elastic critical scattering underneath. As discussed in the text, the inelastic data have been fitted with Eq. (12), convoluted with the instrument resolution, with a narrow Gaussian function included at zero energy transfer for the incoherent scattering. The appropriate fits are also shown in the figures.

of Fig. 8, where $\gamma_{q \sim 0}$ is plotted against the values of $\xi$ at the corresponding temperatures. The values for $\xi$ were calculated from Eq. (7). The data have been fitted with a power law. Expected exponents from scaling theory would be $-3 / 4$ (for the 3DH), -1 (for the CLRM), or -2 (for the 2DAH). Not only are the data not particularly well fitted by a power law, especially for smaller $\xi$, but the value of the exponent $(=-2.6)$ differs considerably from the expected values.

The fitted amplitudes, $A_{q \sim 0}$, also provide useful information, as they should be proportional to the real part of the magnetic susceptibility, $\chi_{q \sim 0}^{\prime} \cdot{ }^{37}$ A number of different instrument configurations were used to measure the data in Fig. 8 and, due to the approximations inherent in estimating the amplitude of the resolution function, it is very difficult to quantitatively compare amplitudes between different configurations. It is possible to compare fits for measurements 


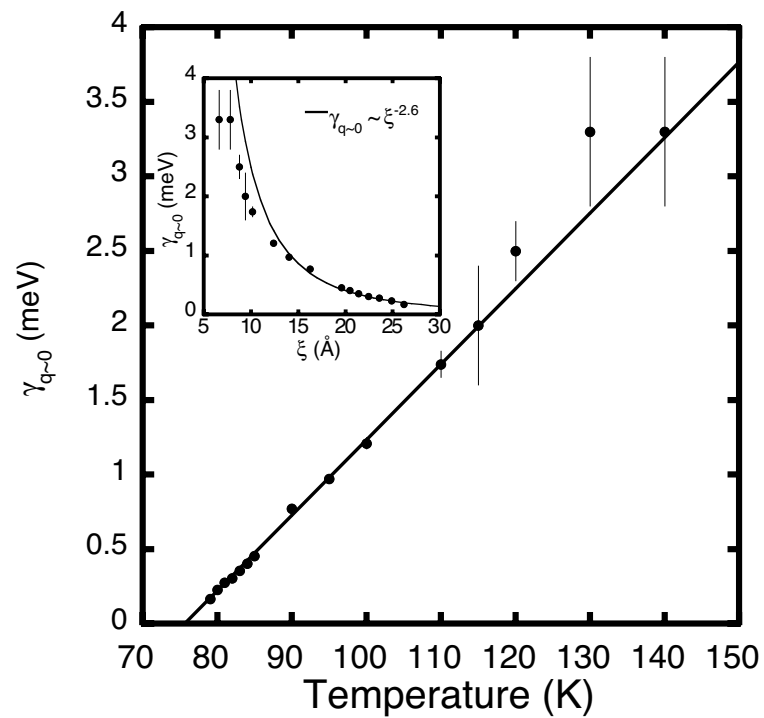

FIG. 8. The variation of the width of the quasi-elastic scattering, $\gamma_{q \sim 0}$, with temperature close to the $(0,2,1)$ Bragg peak. The data have been fitted with a straight line. The inset shows $\gamma_{q \sim 0}$ plotted against the correlation length $\xi$ calculated for each of the temperatures using Eq. (11), along with fits of a power law and an exponential function to the data.

made with the same resolution, and the fitted amplitudes for the configuration that covered the broadest range of temperatures are shown in Fig. 9. The data were fitted with a power law, $\chi_{q \sim 0}^{\prime} \sim\left(T-T_{N}\right)^{-\zeta}$, where $T_{N}$ was the Néel temperature determined experimentally. A Curie-Weiss behavior would

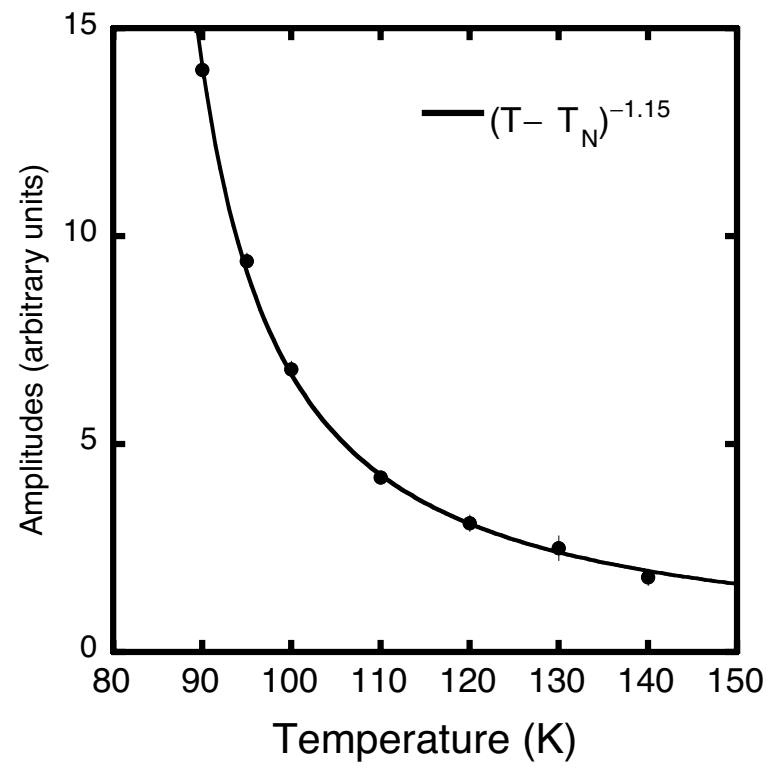

FIG. 9. Some of the amplitudes determined by fitting the quasielastic scattering close to the $(0,2,1)$ Bragg peak above $T_{N}$. The scans were all measured with the same instrument configuration, and therefore the same resolution. The data have been fitted with a power law. The fitted exponent equals -1.15 , and is close to that expected for a Curie-Weiss law. The value for $T_{N}$ used in the fit was that determined experimentally.

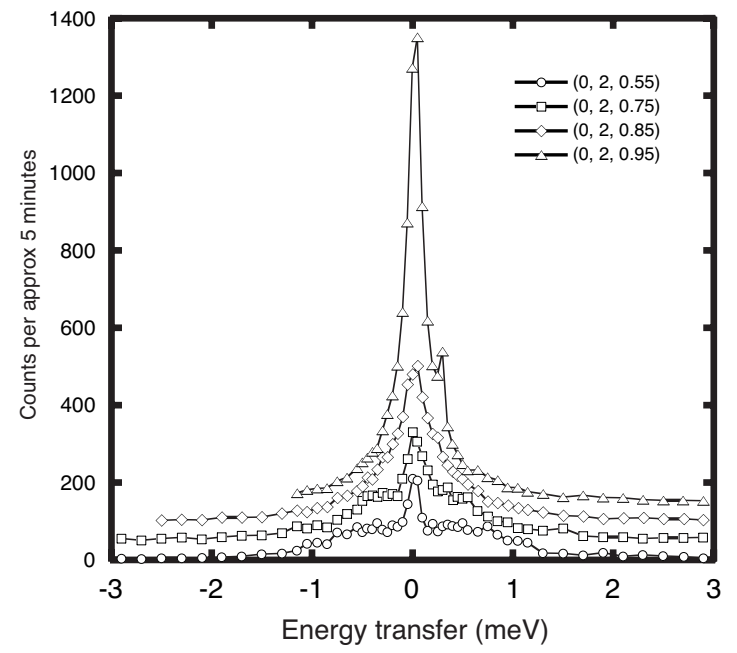

FIG. 10. Measurements of the quasi-elastic scattering along the $c^{*}$ direction at a temperature of $85 \mathrm{~K}$. Each scan has been shifted by 50 vertical units from its neighbors. Note that the Néel temperature in $\mathrm{MnPS}_{3}$ is $\sim 78 \mathrm{~K}$.

have $\zeta=1$, which is close to but outside the error on the fitted exponent, $\zeta=1.15 \pm 0.02$. Departures from Curie-Weiss behavior are common in low-dimensional magnets, and that may explain the small difference.

\section{E. The dynamic critical scattering between the planes}

As previously discussed, at $T_{N}$ the gap in the spin-wave energies goes to zero and the magnetic Bragg peaks in $\mathrm{MnPS}_{3}$ becomes quasielastic with a width $\gamma_{q \sim 0}$. In a $2 \mathrm{D}$ system, this would be true for all the spin waves normal to the planes. $\mathrm{MnPS}_{3}$ does have some exchange between the layer planes, and above $T_{N}$ there is some $q$ dependence in the scattering along the $c^{*}$ direction, as is shown from IN12 measurements in Fig. 10. The scattering along the $c^{*}$ direction was measured to probe the correlation as a function of temperature.

Constant- $Q$ measurements were made at the $(0,2,0.55)$ position, which is close to the Brillouin zone boundary along the $c^{*}$ direction and relatively free of spurious signal including higher-order wavelength contamination. The data at this position could be interpreted either as quasielastic or as heavily overdamped dispersive modes. For consistency of comparison with the analysis at $q \sim 0$, the data were fitted with Eq. (12) to determine the widths $\gamma_{q \sim \text { BZB }}$ that are plotted as a function of temperature in Fig. 11.

Inserted in the figure are examples of the fits to the data at the two lowest-measured temperatures. The data at $79.1 \mathrm{~K}$ is not particularly well represented by a Lorentzian; indeed, an overdamped harmonic oscillator function better fits the data. All the data from $85 \mathrm{~K}$ upwards are, however, very well represented by Eq. (12).

Just above $T_{N}$ the width of the quasielastic scattering close to the Brillouin zone boundary is broader than the width close to the Brillouin zone center. This is consistent with the spin fluctuations between the planes being weakly dispersive, but heavily overdamped. The width $\gamma_{q \sim \text { BZB }}$ as- 


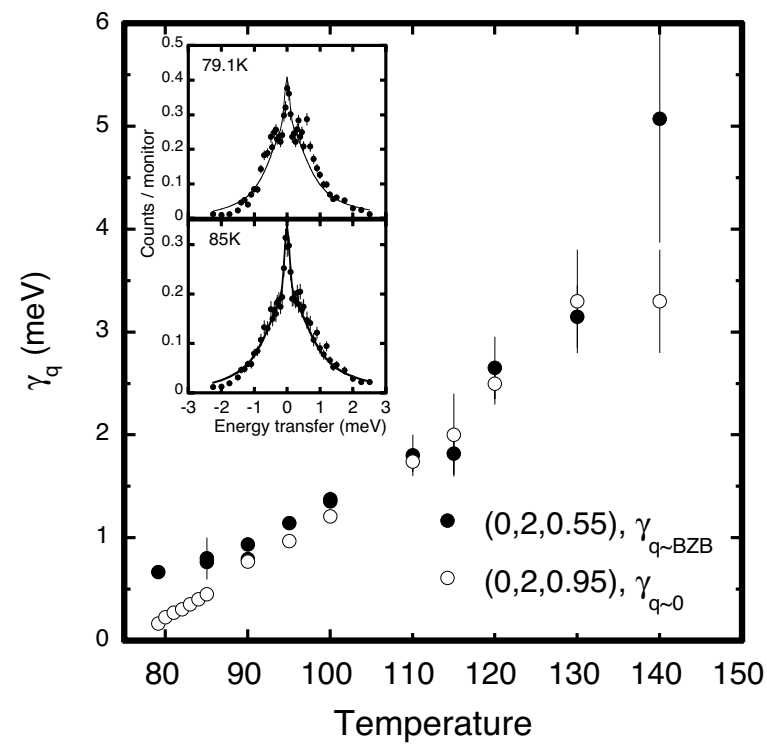

FIG. 11. The widths of the quasielastic scattering along the $c^{*}$ direction as a function of temperature. The widths at $(0,2,0.95)$ are as given in Fig. 8. The widths at $(0,2,0.55)$ result from fitting Eq. (12) to the data. Examples of the fits to the data at $(0,2,0.55)$ are shown in the inset.

ymptotes towards $\gamma_{q \sim 0}$ with increasing temperature and the two meet at $\sim 105 \mathrm{~K}$, above which the two widths are equivalent. The temperature approximately coincides with the maximum of the magnetic susceptibility, ${ }^{7,8}$ and above this temperature it may be assumed that any critical dynamics is completely confined to the $a b$ planes.

\section{F. The dynamical critical scattering within the planes}

The inelastic scattering along $(0, k, 0)$ was also measured above $T_{N}$. Unlike the scattering along the $c^{*}$ direction, the scattering along $(0, k, 0)$ clearly showed dispersive, albeit heavily damped, modes at temperatures well above $T_{N}$. Figure 12 shows examples of the dispersive scattering measured on IN14 at $85 \mathrm{~K}$.

The data were subsequently fitted with the function for a damped harmonic oscillator, developed for the study of anharmonic phonons ${ }^{38}$

$$
S(q, \omega)=\frac{[n(\omega)+1] A_{q} 4 \omega \gamma_{q} / \pi}{\left[\omega^{2}-\Omega_{q}^{2}\right]^{2}+4 \omega^{2} \gamma_{q}^{2}},
$$

where $\Omega_{q}$ is the renormalized frequency at reduced wave vector $q$ and $\gamma_{q}$ is the spin-wave damping. Like Eq. (12), Eq. (13) has the correct form for a dynamic structure factor. The function was again convoluted with the instrument resolution using a Monte Carlo algorithm.

To provide a general and systematically correct fit to all the data measured at one temperature, the renormalized frequency $\Omega_{q}$ was not used as a fit variable. Instead, $\Omega_{q}$ were calculated at each value of $q$ using an expression for the spin-wave dispersion in $\mathrm{MnPS}_{3}$. The full expression requires exchange interactions with up to the third nearest neighbor in plane ${ }^{13}$ however, for small $q$ the expression can be reduced

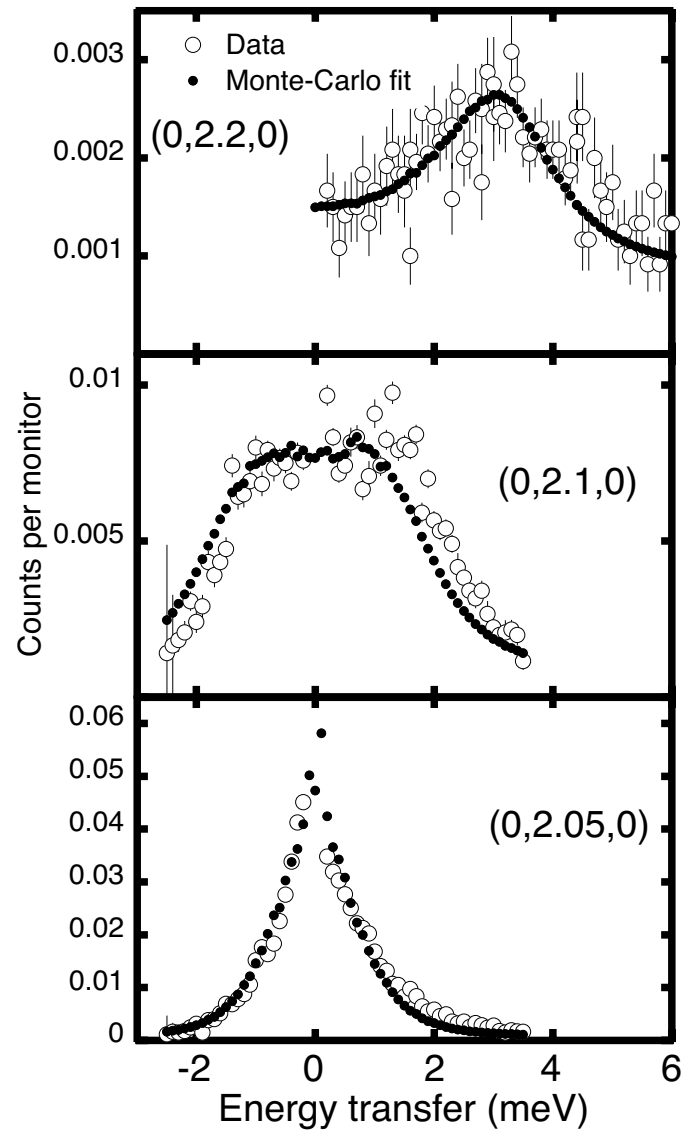

FIG. 12. Measurements of the inelastic scattering along $(0, k, 0)$ at $85 \mathrm{~K}$. The damped, dispersive modes have been fitted with Eq. (13) via a Monte Carlo technique, and the fits are also shown in the figure.

to a function that depends only on the nearest neighbor and the anisotropy, i.e.,

$$
\begin{aligned}
\Omega_{q}^{2} \approx & {\left[g \mu_{B} H_{A}-6 S J_{1}\right]^{2}-\mid 2 S J_{1} \exp \left(-\frac{i 2 \pi}{3} k\right) } \\
& +\left.4 S J_{1} \cos (\pi h) \exp \left(\frac{i \pi}{3} k\right)\right|^{2} .
\end{aligned}
$$

The relevant parameter in fitting the data was therefore $J_{1}$.

Figure 13 shows a comparison between Eq. (14) and the full expression to the ground-state dispersion curve. Most of the dispersion curve can be adequately reproduced using Eq. (14) if $J_{1}=-0.95$. The comparison breaks down close to the Brillouin zone boundary; however, inspection of Fig. 13(b) shows that Eq. (14) is valid for $q \leq 0.2$.

In fitting the data, the anisotropy, $g \mu_{B} H_{A}$, was set to zero, in line with the observation that the gap anisotropy goes to zero at $T_{N \cdot}{ }^{13}$ Initially, $J_{1}$ was allowed to float. It was soon found, however, that all the data for $T>T_{N}$ could be fitted with the exchange interaction fixed to $J_{1}=-0.77 \mathrm{meV}$. The value of $J_{1}$ was then fixed for all fits while $\gamma_{q}$ and $A_{q}$ remained free parameters. Examples of the fits are shown in Fig. 12. Figure 13(b) shows the effect on the dispersion curve of decreasing $J_{1}$ from -0.95 to $-0.77 \mathrm{meV}$. While the 

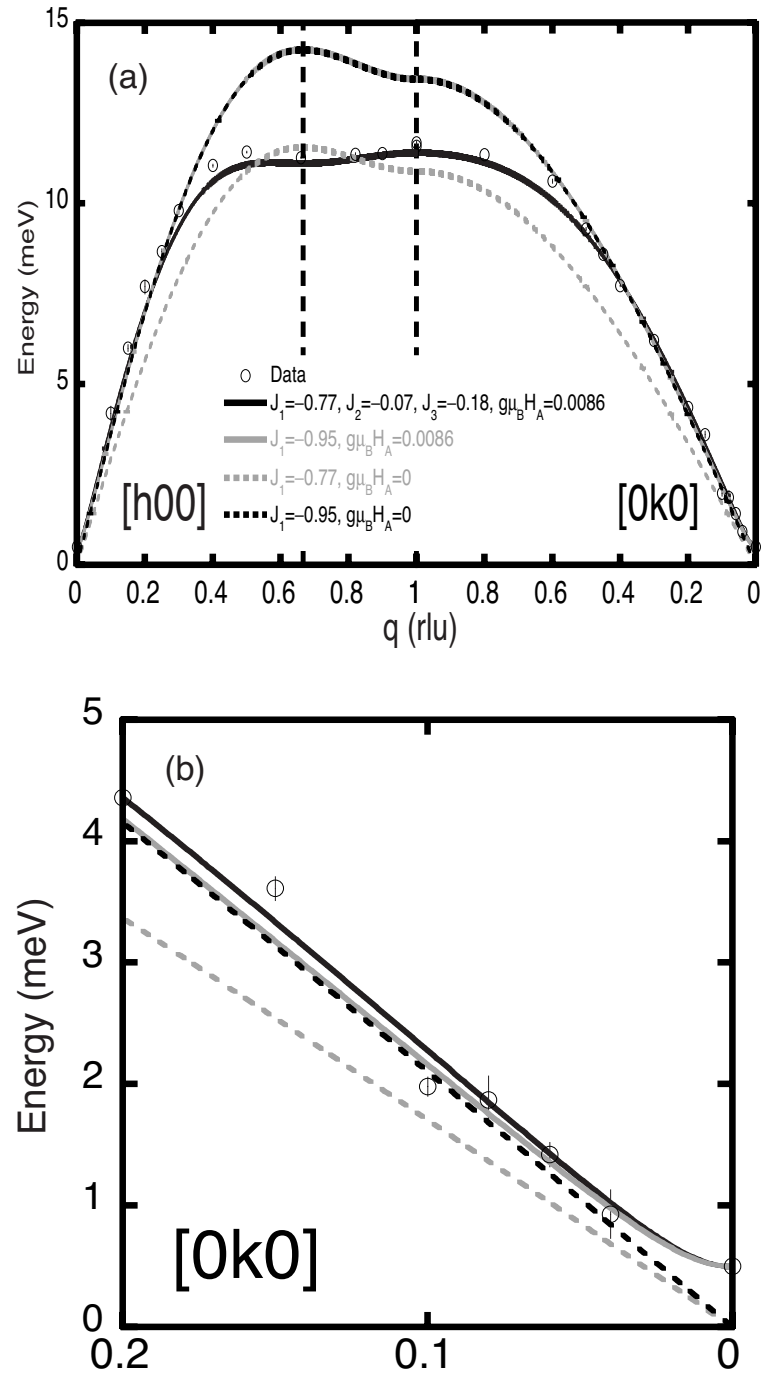

FIG. 13. A comparison between the in-plane spin-wave dispersion curve and models with different exchange parameters. (a) shows the entire Brillouin zone. The best model includes up to the third nearest-neighbor; however, the low- $q$ dispersion may be adequately modeled by using only one exchange parameter with a slightly larger value. Models with no anisotropy are also shown to compare with the spin-wave energies above $T_{N}$. (b) has the small $q$ data along $[0, k, 0]$ on an expanded scale.

spin-wave energies have decreased, the change does not necessarily reflect a renormalization of $J_{1}$, since $-0.77 \mathrm{meV}$ is the size of the nearest neighbor exchange in the ground state. ${ }^{13}$ A better explanation for the change is that, as $T_{N}$ is approached, the interaction between second- and thirdnearest neighbors diminishes. This finding is again at odds with the scaling theory, which predicts a renormalization of the spin-wave stiffness based only on the nearest-neighbor exchange.

The fitted damping parameters $\gamma_{q}$ are shown as a function of temperature and $q$ in Fig. 14(a). The data appear to have some temperature and $q$ dependence, although the two appear to be mutually exclusive. For $T<85 \mathrm{~K}$, the values of $\gamma_{q}$ appear to be constant with $\gamma_{q} \sim 1 \mathrm{meV}$ for all $q$. For $T$ $>85 \mathrm{~K}$, the values of $\gamma_{q}$ remain approximately constant for
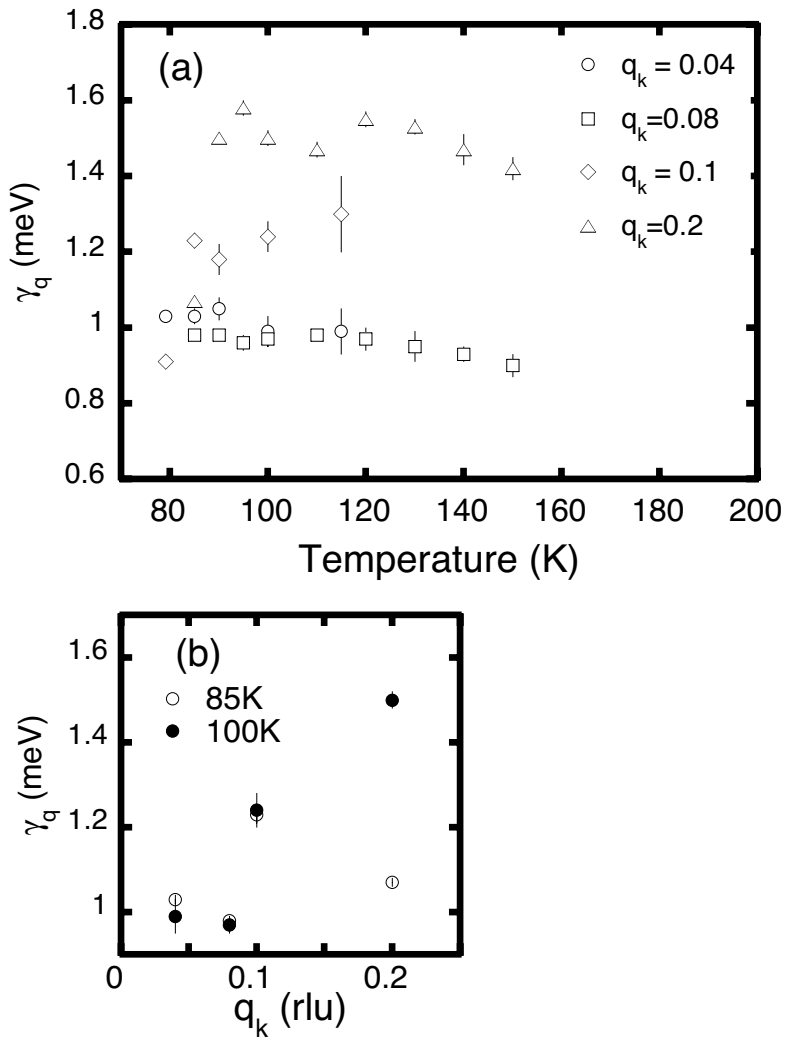

FIG. 14. The fitted spin-wave damping along $(0, k, 0)$ above $T_{N}$. The damping was one of the output parameters from fitting Eq. (13) to the data. (a) shows all the data as functions of temperature. (b) shows two data from two temperatures: one set close to $T_{N}$ where the damping appears to be independent of $q$; and one set at higher temperatures where the damping appears to be $q$ dependent but independent of $T$.

$q \leq 0.08$ rlu with $\gamma_{q \leq 0.08} \sim 1 \mathrm{meV}$. A $q$ dependence is seen, however, for $q>0.08$. The width at $q=0.1$ rises to $\gamma_{q=0.1}$ $\sim 1.2 \mathrm{meV}$ and at $q=0.2$ to $\gamma_{q=0.2} \sim 1.4 \mathrm{meV}$. The widths at all $q$ then remain constant with increasing temperature. Figure 14(b) shows the fitted $\gamma_{q}$ for the range of $q$ at and above $85 \mathrm{~K}$. The $q$ dependence of the data can be compared to the models. All of the models predict that $\gamma_{q}$ should vary with $q$, thus, the data for $T \leq 85 \mathrm{~K}$ cannot be easily explained. Above $85 \mathrm{~K}$, however, comparisons can be made. The CLRM divides reciprocal space into two regimes, each with its own renormalization function. The combined functions show something like a linear dependence in $\gamma_{q}$ at small $q$ and a saturating $\gamma_{q}$ at larger $q .{ }^{19}$ This is contrary to the data in Fig. 14(b), which appear to be approximately constant at small $q$, and then to increase at large $q$. Equation (11) for the $2 \mathrm{DAH}$ does have a functional form that has this behavior. ${ }^{26}$ The equation applies to the width of a quasielastic peak and not to spin waves, which do not appear in the analytical theory. Computer simulations do show spin waves, particularly in the out-of-plane direction. ${ }^{29,31}$ The damping appears to go to zero at small $q$, however, it still has a form which resembles the $q$ dependence of $\gamma_{q}$ for $\mathrm{MnPS}_{3}, T>85 \mathrm{~K}$.

The temperature behavior of $\gamma_{q}$, however, does not seem to correspond exactly to either of the models. The CLRM shows that all $\gamma_{q}$ should increase with increasing 


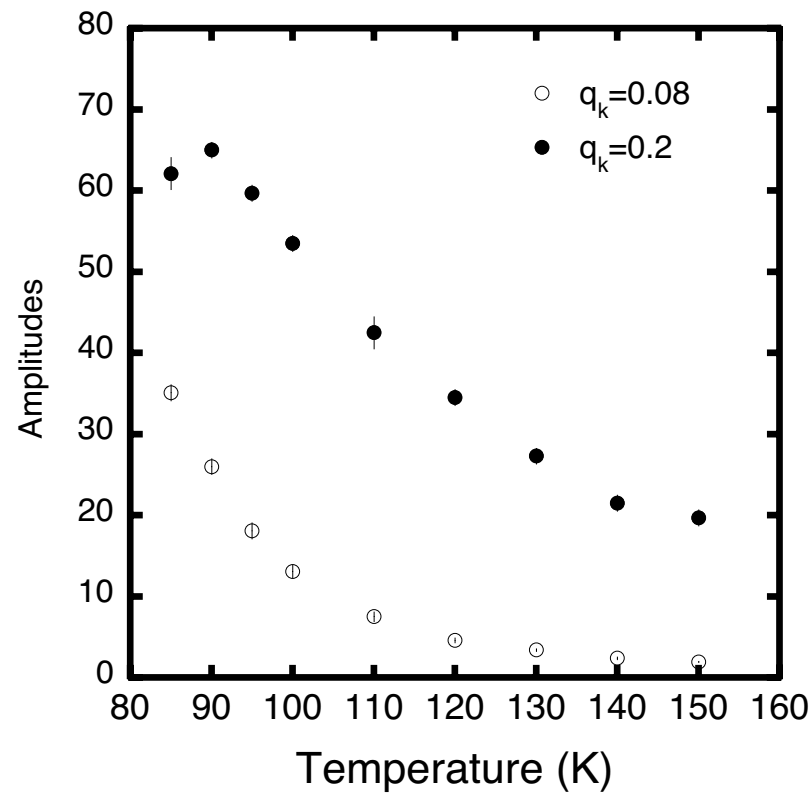

FIG. 15. Some of the amplitudes determined by fitting the data along $(0, k, 0)$ with Eq. (13) above $T_{N}$. The scans were all measured with the same instrument configuration, and therefore the same resolution.

temperature, ${ }^{19}$ which is not observed. Equation (11) for the 2DAH model also predicts an increase; however, this is much smaller and appears to only be at small $q \cdot{ }^{26}$ While this functional form appears to best match the $\gamma_{q}$ data for $T$ $>85 \mathrm{~K}$, computer simulations showing spin waves in the 2DAH show that the damping increases relatively quickly above $T_{K T}$ to the point where the spin waves become a quasielastic central peak. This does not reproduce the observed behavior.

The fitted amplitudes $A_{q}$ from data measured using identical instrument configurations at two values of $q$ are shown in Fig. 15. Unlike the spin stiffness or the damping, the amplitudes show a clear dependence on temperature and on $q$. The amplitudes decrease with increasing temperature and increase with increasing $q$. The dependence of $A_{q}$ on $T$ also appears to be $q$ dependent.

In principle, the amplitudes of the partial cross sections are proportional to $\chi_{q}^{\prime}$. It must be stressed that Eq. (13) is not a cross section and, while it gives a correct functional form for the scattering, there will be $q$-dependent factors in $A_{q}$ that will depend on the Hamiltonian for the system, the exact form of which is open to debate in $\mathrm{MnPS}_{3}$. The fitted widths $\gamma_{q}$ may, therefore, be regarded as quantitative, while the amplitudes should currently be regarded as self-consistent, but qualitative. Figure 15 is thus added for completeness, should there be any future developments in the theory.

\section{DISCUSSION}

The 2DAH model appears to come closest to describing the critical properties of $\mathrm{MnPS}_{3}$ over the entire temperature range, with the exception close to $T_{N}$ where there appears to be a crossover to $3 \mathrm{DH}$ behavior. The sublattice magnetiza- tion below $T_{N}$ and $\xi$ above $T_{N}$ are well described by the static critical behavior of the $2 \mathrm{DAH}$ model, and certain qualitative aspects of the dynamic critical behavior match. This is contrary to the widely held belief that the compound is a good example of a Heisenberg system. The implication is that the Hamiltonian for $\mathrm{MnPS}_{3}$ is not isotropic, but is better described by Eq. (9) for $\lambda<1$. The 2DAH model describes the thermodynamics of vortices, opening the interesting possibility of their presence in $\mathrm{MnPS}_{3}$. The effect of an anisotropic Hamiltonian must be small, however, otherwise the high temperature susceptibility would not be isotropic. ${ }^{7,8}$

The size of $\lambda$ can be estimated. As is seen in Fig. 13, there is a gap in the spin-wave energies of $\mathrm{MnPS}_{3}$ at the Brillouin zone center. This has been modeled by including an anisotropy term in a Heisenberg Hamiltonian with amplitude $g \mu_{B} H_{A} \cdot{ }^{13}$ The parameters $\lambda$ and $g \mu_{B} H_{A}$ are, however, interchangeable for a small anisotropy, linked through the equation $^{25}$

$$
\lambda=1-\frac{g \mu_{B} H_{A}}{4|J|}\left(1-\frac{1}{2 S}\right), \quad \frac{g \mu_{B} H_{A}}{|J|} \ll 1 .
$$

Substituting for the values of $\mathrm{MnPS}_{3}$ gives $\lambda=0.998$. The CLRM will only hold if $\lambda \sim 1$. The small deviation thus puts the Hamiltonian in the same universality class as the 2DXY model. An explanation for why $\lambda$ is not greater than 1 , as might be expected given the orientation of the moments in the ordered state, comes from reports of a second, competing single-ion anisotropy that favors the moments to lie in the $a b$ plane. ${ }^{7,39}$ Dipolar anisotropy is believed to be important in this compound; ${ }^{12}$ particularly as it can explain the fact that in the ordered state the magnetic moments are normal to the $a b$ planes. ${ }^{40}$ The measured anisotropy in $\mathrm{MnPS}_{3}$ is approximately a quarter of the magnitude expected for the dipoledipole component. ${ }^{13,40}$ Competition between the two anisotropies may explain this discrepancy. The dipole-dipole anisotropy determines the moment direction, however, the influence of the second anisotropy, attempting to force the moments in plane, is sufficient to tip the dynamics of the compound to the new universality class. The source of the second anisotropy is currently unclear, and the one outstanding unknown in $\mathrm{MnPS}_{3}$ regards the knowledge of its properties. Were this to be known, a complete theory for the magnetic properties of $\mathrm{MnPS}_{3}$ could be developed.

Some aspects of the critical dynamics can also be explained by the $2 \mathrm{DAH}$ model. While the in-plane dynamical structure factor becomes quasielastic slightly above $T_{K T}$, spin waves can still be present well above $T_{K T}$ if a significant out-of-plane component to the vortices exists. Calculations suggest that a critical value for $\lambda$ in a honeycomb lattice is $\lambda=0.86 .{ }^{30}$ For $\lambda>0.86$, out-of-plane components will dominate the dynamics, an inequality that is consistent with the calculated value of $\lambda=0.998$. In addition, the functional form of Eq. (11) does appear to have a similar $q$ and $T$ dependence to the observed spin-wave damping above $T_{N}$, although it applies to the quasielastic width of the in-plane components.

There are, however, a number of discrepancies regarding the observed critical dynamics. Contrary to any of the models discussed here, the energy widths do not seem to scale 
TABLE I. Comparison of the measured transition temperatures with the calculated values derived from the equations in Ref. 25. The measured $T_{K T}$ is derived from fitting Eq. (7) to the measured correlation length $\xi$ as shown in Fig. 5 .

\begin{tabular}{lccc}
\hline \hline & $T_{K T}(\mathrm{~K})$ & {$\left[T_{K T} /(J S)\right]^{2}$} & $T_{N}(\mathrm{~K})$ \\
Measured & $55 \pm 4$ & 6.06 & 78 \\
\hline Classical: $\left[T_{K T} /(J S)\right]^{2} \gg 32,\left|J^{\prime} / J\right| \ll 1-\lambda$ & 35 & 2.45 & 0 \\
Classical: $\left[T_{K T} /(J S)\right]^{2} \gg 32,\left|J^{\prime} / J\right| \gg 1-\lambda$ & 35 & 2.45 & 186 \\
Quantum: $\left[T_{K T} /(J S)\right]^{2} \ll 32,\left|J^{\prime} / J\right| \ll 1-\lambda$ & 64 & 8.21 & 0 \\
Quantum: $\left[T_{K T} /(J S)\right]^{2} \ll 32,\left|J^{\prime} / J\right| \gg 1-\lambda$ & 64 & 8.21 & 305 \\
\hline \hline
\end{tabular}

with $\xi$, and the spin-wave exchange due to first nearest neighbors does not seem to renormalize with temperature, although the exchange due to second- and third-nearest neighbors in-plane does seem to go to zero above $T_{N}$.

The source of the discrepancies may lie in the fact there is interplanar coupling in $\mathrm{MnPS}_{3}$ that is approximately the same size as the anisotropy, $\left|J^{\prime} / J\right| \sim 1-\lambda$. Irkhin and Katamin have derived expressions giving the transition temperatures for a layered magnet with weak easy-axis anisotropy. ${ }^{25}$ The theory successfully predicts the transition temperatures of a range of systems, and may justifiably be applied to $\mathrm{MnPS}_{3}$. The expressions are derived for two regimes; a classical regime, where $\left[T_{K T} /(J S)\right]^{2} \gg 32$, and a quantum regime where $\left[T_{K T} /(J S)\right]^{2} \ll 32$. The theory further gives a function to calculate the Néel temperature, in the limit of a small interplanar coupling and where $\left|J^{\prime} / J\right|$ is very different from $1-\lambda .{ }^{25}$ The calculated values for the transition temperatures are listed in Table I, as are the actual Néel temperature and the value for $T_{K T}$ determined by fitting Eq. (7) to the data in Fig. 5. The calculated values of $T_{K T}$ differ from the measured value, although the difference is not enormous. Interestingly, the values of $\left[T_{K T} /(J S)\right]^{2}$ are less than 32 . It would, therefore, appear that in the context of this model the properties of $\mathrm{MnPS}_{3}$ correspond to the quantum regime, and indeed the calculated $T_{K T}$ in this regime is closer to the measured value. In general, the agreement is not as close as with other systems. ${ }^{25}$ The calculated Néel temperatures, however, are very different to the actual $T_{N}$. In the case of $\mathrm{MnPS}_{3}$, $\left|J^{\prime} / J\right|=0.0025 \approx 1-\lambda$, thus, the necessary inequalities are not satisfied. The theory said to be qualitatively correct up to $\left|J^{\prime} / J\right| \sim 1-\lambda$ with an appropriate renormalization of a universal constant, $C$. Manipulation of the equations soon shows that no value of $C$ can satisfy both of the measured values $T_{N}$ and $T_{K T}$; the value of $C$ necessary to give $T_{N}=78 \mathrm{~K}$ is $C$ $\sim 6$, which differs greatly from the expected "universal" value $(C \approx-0.5)$ in magnitude and in sign. It is reasonable to assume that theories for the critical dynamics will also have difficulties in the limit $\left|J^{\prime} / J\right| \sim 1-\lambda$. Further theoretical work allowing for an interplanar exchange of similar size to the anisotropy, and aimed specifically at $\mathrm{MnPS}_{3}$, would be useful at this point.

\section{CONCLUSION}

A comprehensive set of data measuring the critical properties of the antiferromagnetic phase transition in $\mathrm{MnPS}_{3}$ has been presented and the results have been discussed in the context of theoretical models. Of the models, the 2D anisotropic Heisenberg model with XY anisotropy appears to be the closest to describing the critical behavior. The exact nature of the anisotropy in the material is obviously the key to a clear understanding of the magnetic properties, which is currently thought to be a competition between dipole-dipole and an in-plane, single-ion anisotropy of unknown origin. That the 2DAH model appears to best describe $\mathrm{MnPS}_{3}$ opens some interesting questions, specifically regarding the possible presence of vortices in the compound. Such vortices are believed to have a very strong out-of-plane component.

\section{ACKNOWLEDGMENTS}

The authors would like to thank the ISIS facility, Ris $\varnothing$ Laboratories, and the Institut Laue-Langevin for providing the beam time and instrumentation. A.R.W. would like to thank Monash University and Trevor Hicks, since much of this article was written while visiting on a sabbatical to that institution. Many thanks also to Roger Cowley and Steven Bramwell for helpful discussion.
*Corresponding author. Electronic address: wildes@ill.fr

${ }^{1}$ L. P. Regnault and J. Rossat-Mignod, in Magnetic Properties of Layered Transition Metal Compounds, edited by L. J. de Jongh (Kluwer Academic, Netherlands, 1990).

${ }^{2}$ N. Rogado, Q. Huang, J. W. Lynn, A. P. Ramirez, D. Huse, and R.

J. Cava, Phys. Rev. B 65, 144443 (2002).

${ }^{3}$ M. Heinrich, H.-A. Krug von Nidda, A. Loidl, N. Rogado, and R.
J. Cava, Phys. Rev. Lett. 91, 137601 (2003).

${ }^{4}$ R. Brec, Solid State Ionics 22, 3 (1986).

${ }^{5}$ V. Grasso and L. Silipigni, Riv. Nuovo Cimento 25, 1 (2002).

${ }^{6}$ G. Ouvrard, R. Brec, and J. Rouxel, Mater. Res. Bull. 20, 1181 (1985).

${ }^{7}$ K. Okuda, K. Kurosawa, S. Saito, M. Honda, Y. Zhihong, and M. Date, J. Phys. Soc. Jpn. 55, 4456 (1986). 
${ }^{8}$ P. A. Joy and S. Vasudevan, Phys. Rev. B 46, 5425 (1992).

${ }^{9}$ N. D. Mermin and H. Wagner, Phys. Rev. Lett. 17, 1133 (1966).

${ }^{10}$ K. Kurosawa, S. Saito, and Y. Yamaguchi, J. Phys. Soc. Jpn. 52, 3919 (1983).

${ }^{11}$ C. Pich and F. Schwabl, J. Magn. Magn. Mater. 140-144, 1709 (1995).

${ }^{12}$ C. Pich and F. Schwabl, J. Magn. Magn. Mater. 148, 30 (1995).

${ }^{13}$ A. R. Wildes, B. Roessli, B. Lebech, and K. W. Godfrey, J. Phys.: Condens. Matter 10, 6417 (1998).

${ }^{14}$ H. M. Rønnow, A. R. Wildes, and S. T. Bramwell, Physica B 276-278, 676 (2000).

${ }^{15}$ P. C. Hohenberg and B. I. Halperin, Rev. Mod. Phys. 49, 435 (1977).

${ }^{16}$ M. F. Collins, Magnetic Critical Scattering (Oxford University Press, Oxford, 1989).

${ }^{17}$ A. Pelissetto and E. Vicari, Phys. Rep. 368, 549 (2002).

${ }^{18}$ S. Chakravarty, B. I. Halperin, and D. R. Nelson, Phys. Rev. B 39, 2344 (1989).

${ }^{19}$ S. Tyč and B. I. Halperin, Phys. Rev. B 42, 2096 (1990).

${ }^{20}$ P. Hasenfratz, Eur. Phys. J. B 13, 11 (2000).

${ }^{21}$ R. J. Christianson, R. L. Leheny, R. J. Birgeneau, and R. W. Erwin, Phys. Rev. B 63, 140401(R) (2001).

${ }^{22}$ Y. S. Lee, M. Greven, B. O. Wells, R. J. Birgeneau, and G. Shirane, Eur. Phys. J. B 5, 15 (1998).

${ }^{23}$ S. T. Bramwell and P. C. W. Holdsworth, J. Phys.: Condens. Matter 5, L53 (1993).

${ }^{24}$ J. M. Kosterlitz, J. Phys. C 7, 1046 (1974).

${ }^{25}$ V. Yu. Irkhin and A. A. Katanin, Phys. Rev. B 60, 2990 (1999).
${ }^{26}$ F. G. Mertens, A. R. Bishop, G. M. Wysin, and C. Kawabata, Phys. Rev. B 39, 591 (1989).

${ }^{27}$ D. G. Wiesler, H. Zabel, and S. M. Shapiro, Z. Phys. B: Condens. Matter 93, 277 (1994).

${ }^{28}$ H. G. Evertz and D. P. Landau, Phys. Rev. B 54, 12302 (1996).

${ }^{29}$ G. M. Wysin, M. E. Gouvêa, and A. S. T. Pires, Phys. Rev. B 62, 11585 (2000).

${ }^{30}$ M. E. Gouvêa, G. M. Wysin, A. R. Bishop, and F. G. Mertens, Phys. Rev. B 39, 11840 (1989).

${ }^{31}$ B. V. Costa, J. E. R. Costa, and D. P. Landau, J. Appl. Phys. 81, 5746 (1997).

${ }^{32}$ A. R. Wildes, M. J. Harris, and K. W. Godfrey, J. Magn. Magn. Mater. 177-181, 143 (1998).

${ }^{33}$ G. Ouvrard and R. Brec, Eur. J. Solid State Inorg. Chem. 27, 477 (1990).

${ }^{34}$ K. Nagata and Y. Tomono, J. Phys. Soc. Jpn. 36, 78 (1974).

${ }^{35}$ C. M. J. van Uijen and H. W. de Wijn, Phys. Rev. B 30, 5265 (1984).

${ }^{36}$ F. Keffer, Handbuch der Physik (Springer, Berlin, 1966), Vol. 18II, p. 68.

${ }^{37}$ S. W. Lovesey, Theory of Neutron Scattering from Condensed Matter (Clarendon Press, Oxford, 1986), Vol. 2.

${ }^{38}$ B. Fåk and B. Dorner, Physica B 234-236, 1107 (1997).

${ }^{39}$ D. A. Cleary, A. H. Francis, and E. Lifshitz, Chem. Phys. 106, 123 (1986).

${ }^{40}$ D. J. Goossens, A. R. Wildes, C. Ritter, and T. J. Hicks, J. Phys.: Condens. Matter 12, 1845 (2000). 